\title{
A negative D-dimer test result alone or combined with low risk clinical status effectively ruled out symptomatic DVT
}

Lennox AF,Delis KT, Serunkuma S, et al. Combination of a clinical risk assessment score and rapid whole blood D-dimer testing in the diagnosis of deep vein thrombosis in symptomatic patients.J Vasc Surg 1999 Nov;30:794-804.

QUESTION: Can a D-dimer assay, alone or combined with clinical examination results, rule out all, proximal, and calf deep venous thrombosis (DVT) in symptomatic patients?

\section{Design}

A blinded comparison of D-dimer test results, either alone or combined with a clinical risk assessment score, with duplex ultrasonographic scanning (DUS).

\section{Setting}

A university hospital in London, United Kingdom.

\section{Patients}

200 consecutive inpatients and outpatients (mean age 58 y, age range 18 to 91 y, $63 \%$ women, $59 \%$ inpatients) with suspected DVT. Exclusion criteria were previous or chronic DVT, symptom duration $>1$ month, anticoagulant therapy $>48$ hours before assessment, or suspected or confirmed pulmonary embolism.

\section{Description of tests and diagnostic standard} Patients were classified as being at low, moderate, or high risk for DVT based on a clinical assessment score that included data on the presence of cancer, immobilisation, localised tenderness or leg swelling, family history, history of leg trauma, unilateral pitting oedema or erythema, dilated superficial veins, hospitalisation within 6 months, and erythema of symptomatic leg only. D-dimer levels were assessed by using a rapid whole blood assay (SimpliRED, Agen Biomedical, Brisbane, Australia) by an examiner who was blinded to the clinical findings. DUS (diagnostic standard) was done in a

blinded manner to identify all, proximal, and calf DVT. Patients with inconclusive scan results had repeated scans done until the diagnosis was conclusive.

\section{Main outcome measures}

Sensitivity and specificity for the diagnosis of DVT for patients with varying risk based on clinical assessment.

\section{Main results}

Of the 200 patients assessed, 44\% were at low risk for DVT. $14 \%$ of patients had proximal DVT, and 9\% had isolated calf DVT. The sensitivity of D-dimer assay was $100 \%$ in detecting proximal DVT and $91 \%$ for all DVT (table).

\section{Conclusion}

A negative D-dimer test result, alone or combined with low risk status, effectively ruled out (high sensitivity) all and proximal DVT in symptomatic people.

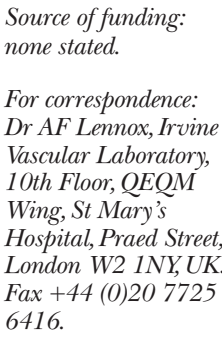

D-dimer assay in the diagnosis of symptomatic deep venous thrombosis (DVT)

\begin{tabular}{llrlll} 
Patients & DVT & Sensitivity & Specificity & +LR & -LR \\
All & Proximal & $100 \%$ & $76 \%$ & 4.2 & 0.0 \\
\hline All & Isolated calf & $82 \%$ & $69 \%$ & 2.7 & 0.3 \\
\hline All & All & $91 \%$ & $82 \%$ & 5.1 & 0.1 \\
\hline High risk & All & $100 \%$ & $47 \%$ & 1.9 & 0.0 \\
\hline Moderate risk & All & $75 \%$ & $78 \%$ & 3.4 & 0.3 \\
\hline Low risk & All & $75 \%$ & $90 \%$ & 7.5 & 0.3 \\
\hline
\end{tabular}

*LRs defined in glossary and calculated from data in article.

\section{COMMENTARY—continued from previous page}

not be as accurate in settings outside the institutions involved in these and similar studies. Thus, the study results that pertain to calf DVT should be interpreted with caution.

When the study results were limited to proximal DVT, the combination of a negative D-dimer test result and a low clinical likelihood effectively excluded proximal DVT in about $99 \%$ of patients. Another noteworthy finding is that the D-dimer test is not sufficiently accurate to be used as a "stand alone" test in patients with suspected DVT. Although Lennox and colleagues reported a sensitivity of $100 \%$ with D-dimer alone for proximal DVT, this finding is based on relatively few patients. In the study by Aschwanden and colleagues, the sensitivity of $\mathrm{D}$-dimer for proximal DVT, $89 \%$, was lower than that reported in other studies ${ }^{4}$ and may reflect a higher proportion of patients with less extensive DVT and the inclusion of patients with previous DVT. Patients with previous DVT may have a persistently abnormal DUS in the absence of acute DVT.

Clinicians should be aware that D-dimer tests may show false negative results in patients with small popliteal DVT or calf DVT and in patients with biochemi-

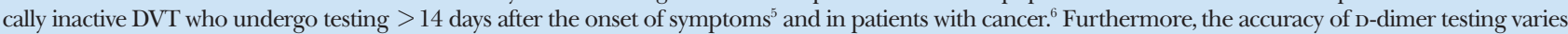
depending on the assay used. ${ }^{4}$ Thus, a structured history and clinical examination is an integral component in the evaluation of patients with suspected DVT.

Are the results of these studies sufficient to change current clinical practice? Not yet. Before DUS can be rendered unnecessary in patients with suspected DVT who have a low clinical likelihood and a negative D-dimer test result, prospective studies with important clinical outcomes are needed to validate the safety of this management approach. Additional research questions include the clinical utility of D-dimer testing in patients with suspected recurrent DVT and in patients who are receiving anticoagulant therapy.

1 Bernardi E, Prandoni P, Lensing AW, et al. Ultrasound Study Investigators Group. BMJ 1998;317:1037-40.

2 Ginsberg JS, Kearon C, Douketis J, et al. Arch Intern Med 1997;157:1077-8.

3 Wells PS, Anderson DR, Bormanis J, et al. Lancet 1997;350:1795-8.

4 Becker DM, Philbrick JT, Bachhuber TL, et al. Arch Intern Med 1996;156:939-46.

5 Turkstra F, van Beek EJ, Buller HR. Thromb Haemost 1998;79:91-3.

6 Lee AY, Julian JA, Levine MN, et al. Ann Intern Med 1999;131:417-23. 